\title{
Progress in gene therapy for breast cancer and what comes next?
}

Bottai Giulia, MS, ${ }^{1}$ Truffi Marta, $\mathrm{PhD},{ }^{2}$ Corsi Fabio, MD, ${ }^{2,3}$ Santarpia Libero, MD, $\mathrm{PhD}^{1}$

${ }^{1}$ Experimental Therapeutics Unit, IRCCS Clinical and Research Institute Humanitas, Via Manzoni 113 - 20089 Milan, Italy

${ }^{2}$ Department of Biomedical and Clinical Sciences "L. Sacco", University of Milan, via G. B. Grassi 74 - 20157 Milan, Italy

* Corresponding authors:

Libero Santarpia, MD, PhD - Experimental Therapeutics Unit, IRCCS Humanitas Clinical and Research Institute, Via Manzoni 56 - 20089 Milan, Italy, Phone: +39 028224 5173; Fax: +39 02 8224 5191, e-mail: libero.santarpia@humanitasresearch.it or liberosantarpia@yahoo.it

Fabio Corsi, MD - Department of Biomedical and Clinical Sciences "L. Sacco", University of Milan, Via G. B. Grassi, 74 - 20157 Milan, Italy, Phone: +39 0250319850-19858, e-mail: fabio.corsi@unimi.it 


\begin{abstract}
Introduction: The possibility of correcting defective genes and modulating gene expression through gene therapy has emerged as a promising treatment strategy for breast cancer. Furthermore, the relevance of tumor immune microenvironment in supporting the oncogenic process has paved the way for novel immunomodulatory applications of gene therapy.

Areas covered: In this review, we describe the most relevant delivery systems, focusing on nonviral vectors, along with the description of the major approaches used to modify target cells, including gene transfer, RNA interference (RNAi), and epigenetic regulation. Furthermore, we highlight innovative therapeutic approaches, and the application of gene therapy in clinical trials for breast cancer.

Expert opinion: Gene therapy has the potential to impact clinical research. However, the safety and efficacy profile of delivery systems, and the specificity of gene modification must be improved. Further efforts are required to increase the clinical application of RNAi-based therapeutics, especially in combination with conventional treatments. Innovative strategies, including genome editing and stem cell-based systems, may certainly contribute to translate gene therapy into clinical practice. Importantly, immune-based strategies emerged as an attractive therapeutic opportunity for selected breast cancer patients. However, several technical challenges need to be addressed before using gene therapy as a concrete option for the treatment of breast cancer patients.
\end{abstract}

Keywords: breast cancer, gene therapy, nanovectors, immunotherapy, vaccine, clinical trials 


\section{Introduction}

Breast cancer is the most frequently diagnosed cancer and a major cause of cancer death in women worldwide. Overall, breast cancer represents a complex and heterogeneous disease, encompassing several distinct entities with different molecular features and clinical behavior. Distinct genetic alterations and gene expression profiles differentially affect the development and progression of breast cancer subtypes, as well as individual patients' outcome and response to treatment. In this context, the possibility of correcting defective genes and modulating gene expression through gene therapy has emerged as a promising treatment strategy for breast cancer $[1,2]$. The genetic modification of a target cells can be reached transferring genes, segments of genes, or oligonucleotides, including small-interfering RNAs (siRNAs) and microRNAs (miRNAs), by in vivo or ex vivo approaches [2]. Furthermore, the therapeutic potential of several genome editing techniques, such as zinc-finger nucleases (ZFNs), transcription activator-like effector nucleases (TALENs) and clustered regularly interspaced short palindromic repeat (CRISPR)-associated protein 9 (Cas9) systems, has been explored [2, 3]. Numerous strategies have been employed to transport the genetic materials to cancer cells, including viral and non-viral vectors $[4,5]$. With the advancement of gene delivery techniques, several gene therapy clinical trials have been performed and are currently ongoing for cancer [2, 5-7]. However, the success of gene therapy can be limited by the heterogeneity between and within tumors, the inability to fully overcome the cellular and tissue barriers to deliver the therapeutic molecule, and the ineffectiveness to transform a sufficient number of cancer cells with the new genetic information. To enhance the therapeutic potential of gene therapy, combinatorial approaches with targeted- or chemotherapeutic- agents have been proposed $[8,9]$.

Besides genetic mutations and gene expression deregulation, emerging evidence suggests that the oncogenic process is consistently supported by the cancer microenvironment, especially by a defective tumor immune response [10]. These findings pave the way for novel application of gene 
therapy, including the immunomodulation of cancer cells and the host immune system, and the delivery of factors that boost anti-tumor immunity.

\section{Delivery technologies for human gene therapy}

Historically, the fundamental limitation for in vivo clinical application of gene therapy is the lack of effective and safe delivery systems, which allow protection of the therapeutic molecules, efficient and specific uptake by the tumor, and ability of crossing biological barriers such as blood vessels and extracellular matrix. Several research and clinical studies have employed viral and non-viral vectors for the systemic delivery of the therapeutic molecules. However, both strategies present several limitations, and improvements of the efficiency and safety of delivery technologies are required prior to clinical application.

\subsection{Virus-based approaches}

To provide effective gene therapy, researchers have first evaluated strategies based on virus infection, which recapitulates a naturally occurring process of genetic intervention in mammalian cells. As a consequence, viral-based approaches were implemented as delivery carriers for nucleic acids in genetic disorders, and then as alternative treatment in non-Mendelian diseases such as cancer $[6,11,12]$. Viral vectors mainly rely on retrovirus, adenovirus, and adeno-associated viruses that were engineered to avoid viral replication and to replace viral DNA with the gene of interest. Therefore, host cells infection results in the introduction of exogenous genetic material, with transient or stable alteration of the genetic heritage of the cell $[6,11]$. In particular, oncolytic viruses, such as herpes simplex virus type $1(\mathrm{HSV}-1)$, have been genetically engineered and explored for their anti-cancer therapeutic potential. They present cytotoxic effects coupled with a tropism for cancer cells, thus causing direct and specific destruction of malignant cells but also stimulating the host immune response $[11,12]$. Despite clinical trials have shown relatively high transfection efficiency and beneficial results in a fraction of patients, the clinical use of viral vectors 
has often been severely limited by intrinsic disadvantages, including insertional mutagenesis, small cargo capacity, inability to reach inaccessible tumors due to loco-regional administration $[12,13]$. Importantly, virus-based approaches raised some safety issues, leading to reconsideration of the clinical application of viral vectors, thus accelerating the development of alternative non-viral carriers.

\subsection{Non-viral nanovectors}

Facing the huge potential of gene therapy in cancer, non-viral systems have been studied and developed to optimize DNA and RNA transfection of cancer cells. Non-viral strategies exploited for gene delivery include transduction of naked nucleic acids favored by physical intervention on cell permeability or provided through the aid of chemical compounds [2, 4, 14]. In particular, nanotechnology has revealed great potential in both increasing efficacy and reducing toxicity of chemotherapy in preclinical and clinical studies of breast cancer $[15,16]$. Furthermore, nanovectors have been explored for breast cancer gene therapy, including RNA interference (RNAi) approaches, suicide genes delivery, and generation of nano-platforms for the co-delivery of chemotherapeutics and RNA molecules [17-20]. A schematic overview of non-viral systems, different cargos and functionalization strategies is shown in Figure 1.

Advances in using nanoparticles as gene delivery vectors are based on the possibility to develop various types of carriers for improving the pharmacokinetic and pharmacodynamic profiles of oligonucleotides, which usually display short half-life and rapid clearance, overcoming undesirable toxicity and immunogenicity, and enabling cellular membrane crossing and tumor penetration $[2,21]$. The packaging of single or double stranded oligonucleotides or plasmids into nanoparticles (of less than $200 \mathrm{~nm}$ in size) facilitates the intratumor accumulation of the therapeutic agents due to enhanced permeability and retention effect (EPR), a phenomenon of passive crossing through the leaky tumor vasculature [21]. Moreover, several strategies for nanoparticles bioengineering have allowed the conjugation of targeting moieties onto the surface of nanoparticles 
in order to increase tumor selectivity and the specific uptake in target tumor cells. This active targeting approach allows exclusive recognition, entrance and release of the therapeutic agent only in target cells, thus achieving effective blockade of cancer progression, while minimizing side effects in healthy cells and tissues [16]. Conventional nanomaterials used to deliver gene-silencing agents include lipid, polymer and inorganic nanoparticles, but different innovative nanoarchitectures are now being explored to increase efficacy.

\subsubsection{Lipid-based nanovectors}

Classical transfection systems for mammalian cells are based on cationic lipid formulations, which confer stability and favor the uptake of negatively-charged nucleic acids. Accordingly, most of the candidate non-viral anti-cancer gene therapeutic strategies involve formulation into lipid-based nanovectors, which include liposomes, stable nucleic acid lipid particles (SNALPs) and lipidoid nanoparticles $[2,22]$. Gene silencing products with lipid nanoparticles have been widely exploited for siRNA delivery. Indeed, some lipid-based nanoparticles are currently in clinical trial, and novel cationic lipid molecules have been proposed for their ability to increase effectiveness [2, 20, 22-25]. Among them, SNALPs, made of ionizable cationic lipids as a core component, display huge siRNA encapsulation capability, together with the maintenance of uniform and small size. SNALPs composed of different lipid formulations have been developed for breast cancer therapy. These systems can package siRNAs directed toward key tumorigenic genes, such as polo like kinase 1 (PLK1), human protein-kinase N3 (PKN3), and $\beta 3$ integrin $[20,25,26]$. In preclinical studies, effective suppression of tumor growth and onset of metastasis in mice bearing orthotopic xenografts of HER2-positive or triple negative breast cancer (TNBC) have been demonstrated [25, 27].

\subsubsection{Polymer-based nanovectors}

Non-lipid nanovectors contain chitosan, polyethylenimines (PEI), dendrimers, poly(lactic-coglycolic acid) and other polymeric materials used as building blocks. Similarly, to most lipid-based 
nanovectors, they generally function as polycation systems to induce the formation of nanosized complexes by electrostatic interactions with negatively charged nucleic acids [2]. Some cationic polymers have been extensively studied in recent years due to their versatility in structure and assembly, which allows efficient delivery, protection and intracellular release of nucleic acids [20, 26]. The strong positive charge of these vectors increases nucleic acid stability on one side, but can also promote colloidal instability of the final complex in physiological condition, inducing undesirable side effects such as increased cellular toxicity and non-specific accumulation in vivo. Therefore, modifications of these polymer-based complexes with hydrophilic polyethylene glycol (PEG), hydrogels, aliphatic lipids or small hydrophobic substitutions have been implemented to enhance colloidal stability of the complex, and improve biocompatibility and blood circulation time upon administration in vivo [28].

Achievement of complex stability is particularly relevant to develop vectors for RNA coupling. Indeed, siRNAs and miRNAs hold some unfavorable features, such as small molecular size and high rigidity, which hinder complex stability [29]. In this regard, the choice of the appropriate nanovector (i.e. molecular weight, structure, surface modification and charge) is essential for achieving therapeutic efficacy. To improve the in vivo delivery of therapeutic nucleic acids, combination of cationic polymer-based complexes with lipids has been deeply explored. This process involves the non-covalent modification of PEI polyplexes with phospholipids to obtain the innovative lipopolyplexes [28]. For their formation, the usage of neutral, anionic or PEG-coated phospholipids is suitable to reduce surface charges, and enhance transfection efficiency and colloidal stability. In particular, lipid-polymer nanoparticles have been evaluated as drugs delivery systems and for the treatment of multi-drug resistant breast cancer [30]

\subsubsection{Inorganic nanoparticles}

Different classes of inorganic nanoparticles have been explored as gene vectors due to their ability to promote oligonucleotides adsorption, concentration and protection [14]. Metallic nanoparticles, 
carbon nanotubes, mesoporous silica and calcium phosphate nanoparticles are included in this category. Compared with lipid-based vectors, dendrimers or biodegradable polymers, inorganic nanoparticles present advantageous features, including high colloidal stability, tunable size and shape, and ease of surface functionalization [14]. However, their clinical application requires biocompatibility and the absence of antigenic activation, which is usually achieved by creating hybrid nanosystems with surfactants coating or lipid modifications. Genes can be loaded onto positively charged coatings, encapsulated into calcium phosphate nanoparticles by means of electrostatic interactions, or conjugated to cleavable linkers onto the nanoparticle surface [31-33].

Intrinsic properties of some inorganic materials can contribute to effectiveness of anti-cancer treatments. In case of magnetic nanoparticles, cancer cells transfection can be accelerated by applying an external magnetic field, which promotes phagocytosis of the genetic nano-agent through a process known as magnetofection [34]. Gold nanoparticles and nanorods are instead investigated for photothermal gene delivery, in which electrostatically bound DNA can be released from the complex under laser radiation without disruption of the genetic material $[32,35]$. In recent years, calcium phosphate nanoparticles have been intensively reconsidered in DNA/RNA delivery for breast cancer therapy [31, 33]. As other cations, they can form stable ionic complexes with a considerable payload of nucleic acids, and possess high transfection capability and easy biodegradation. Major concerns that limited the use of these nanoparticles have been overcome by the coupling with polymeric systems, PEG or lipid shells, reducing aggregation and increasing nanoparticle stealth and cellular penetration [31-33]. Different strategies for inorganic nanoparticles functionalization with aptamers or other targeting ligands have greatly improved the selective targeting of breast cancer cells and transfection efficiency [17].

\subsubsection{Oligonucleotide nanotechnology}

In addition to the above-described nanovectors, nanosystems made of free nucleic acids as building blocks have also been proposed in some breast cancer settings with promising results [36]. They 
consist of nanometer-scale architectures constructed via bottom-up self-assembly of DNA fragments and therapeutic RNA. In this class of nanosystems, the size of the complex, as well as the spatial orientation and density of cancer-targeting ligands on the nanoparticle surface can be finely controlled. Moreover, the sequence of RNA nanoparticles can be tuned to either induce an inflammatory response in the tumor microenvironment or achieve absence of immunogenicity [37, 38].

\section{Gene therapeutic strategies}

The wide range of delivery technologies now available have been explored in order to vehicle several classes of gene-based therapeutic agents. As distinct genetic alterations and gene expression profiles differentially affect the development and progression of breast cancer, correction of defective genes and regulation of gene expression through gene therapy has emerged as an innovative treatment strategy for breast cancer. Several approaches to induce genetic modification of a target cell have been evaluated, including transferring genes, segments of genes, or oligonucleotides such as siRNAs and miRNAs.

\subsection{Gene replacement and gene addition}

The more classical view in gene therapy for cancer and other diseases was represented by the replacement of mutated genes with their normal counterparts. Indeed, loss-of-function mutations in tumor suppressor genes have been identified as key events in breast cancer, with subsequent uncontrolled tumor progression and onset of metastasis. Therefore, the transfection of cancer cells with completely functional tumor suppressor genes (e.g. TP53) has been investigated as an anticancer strategy with both viral vectors and few nanosystems, resulting in inhibition of breast tumor growth and apoptosis induction [39, 40]. To obtain the death or growth arrest of cancer cells, another investigated strategy regards the transfection of suicide genes coding for toxins or drugactivating enzymes that convert prodrugs into cytotoxic compounds. In particular, the transfer of the 
HSV-mediated thymidine kinase (TK) gene in combination with gancyclovir treatment, or the use of cytosine deaminase (CD) gene under the control of the HER2 promoter to induce breast tumor reduction upon conversion of the prodrug fluorocytosine into 5-fluorouracil (5-FU), have been successfully investigated in breast cancer clinical trials [18].

A quite distinct conceptual approach aims at reducing the severe toxic effects of chemotherapy, by promoting bone marrow protection. The transfer of drug resistant genes in hematopoietic stem cells ex vivo and subsequent autologous transplantation of transfected cells in patients will make chemotherapy more effective and tolerated even at higher dosage [41].

\subsection{Alteration of gene expression}

Beyond gene addition, silencing of gene expression is another therapeutic strategy that has revealed impressive impact on cancer therapy. The possibility to shut down oncogenes and key drivers involved in cancer initiation, progression, metastasis and angiogenesis by transfecting antisense oligonucleotides with specific sequence, has greatly contributed to increase the potential of cancertargeted therapy (Table 1).

Since the discovery of the RNAi as a key post-transcriptional mechanism of gene expression regulation, siRNAs and short hairpin RNAs (shRNAs) have gained interest [22]. Their mechanism of function is perfect matching to target gene transcript and subsequent prevention of protein translation with high specificity. The ability of RNAi systems to virtually target any oncogenic process greatly enlarges the potential of targeted therapies, which was strictly limited to classical “druggable" targets. A number of siRNA targets are attractive for breast cancer treatment, including cell cycle regulators, signaling proteins, proto-oncogenes, players of epithelial-to-mesenchymal transition (EMT), angiogenic factors, and proteins associated with drug resistance. An overview of the most recent RNAi-based strategies evaluated in preclinical models of breast cancer is shown in Table 1. Over time, the identification of effective antisense and RNAi drugs has faced several challenges including toxicity, sensitivity to degradation, susceptibility to dissociation after systemic 
injection, low cellular uptake, and lack of specificity. These challenges have been largely overcome by the introduction of modifications in the backbone of the oligonucleotides, making them chemically stable and less susceptible to degradation. Even though delivery systems still represent a limitation, the effectiveness of siRNA therapies has been extensively demonstrated, and some nanovectors have shown outstanding advantages for the delivery of these molecules $[2,8]$.

\subsection{Epigenetic regulation}

Although some genes have been discovered as key drivers of cancer initiation and progression, and targeted therapies have revealed impressive efficacy in tumor management, it is worth noting that targeting a single gene may be an inefficient long-term treatment strategy due to genetic instability and intrinsic heterogeneity of cancers. For this reason, another class of RNA molecules has drawn particular interest in the field of gene silencing and gene expression regulation (Table 1).

MiRNAs are single-stranded RNAs that promote gene expression regulation at the posttranscriptional level, by binding the target transcripts and repressing translation [73]. In both physiological and disease settings, these molecules play crucial roles in regulating cell cycle, proliferation, differentiation, metabolism and apoptosis [73]. Accordingly, the expression level of many miRNAs has been found altered in tissue and/or plasma of breast cancer patients [74-77]. Deregulation of miRNA levels has important implications in cancer, because a single miRNA can simultaneously affect a broad set of targets. Therefore, both miRNA mimics and miRNA inhibitors have been developed and successfully tested as therapeutic agents to achieve breast tumor regression. In particular, miRNA-based therapeutic strategies are now extensively explored in breast cancer preclinical models in order to regulate the expression of oncogenic genes and pathways and the interaction with the immune microenvironment, to modify the expression of surface markers, and to restore cancer cells sensitivity to therapy [33, 36, 42, 44, 62, 71, 74, 78]. This is particularly relevant for aggressive cancers that still lack effective drugs, such as TNBC, or in case of onset of resistance. The ability to selectively manipulate tumors and restore the cellular 
equilibrium of deregulated miRNA expression profiles is now emerging as a promising clinical opportunity thanks to the impressive improvements in the development of non-viral nanocarriers, including the combination of miRNA-based therapy with other anticancer therapies, and the exploration of potential synergistic effects on tumor growth [8].

\section{Prospective advancements for gene therapy in breast cancer}

Anti-cancer treatments have several major limitations, including severe side effects and the emergence of drug resistance. The use of combination therapy involving gene therapy and agents that are currently the standard of care for breast cancer has been suggested as an innovative therapeutic strategy. However, conventional DNA- and RNA-based gene therapies pose great safety and efficiency challenges due to the risk of mutagenesis, delivery barriers, and transient and offtargets effects. Thus, further efforts are needed to identify and develop more effective systems to modify cancer-related genes and deregulated downstream pathways. Human mesenchymal stem cells (MSCs) have been proposed as potential innovative vehicles for gene therapy due to their selfrenewing and tumor-homing ability, and low immunogenicity. Furthermore, advancement in genome engineering technologies, including the development of CRISPR/Cas9 systems has the potential to revolutionize clinical research and personalized treatments. Besides genetic mutations and gene expression aberrations, the host immune anti-cancer response has been deeply investigated for immunotherapy applications. Genetic engineering of autologous $\mathrm{T}$ lymphocytes or dendritic cells (DCs), and cancer vaccines represent powerful strategies to generate a specific anti-tumor immune response against a patient's cancer.

\subsection{Combinatorial treatments}

Treatment strategies in breast cancer are based on clinicopathological and molecular classification [79]. While hormonal therapy and anti-HER2 monoclonal antibodies have substantially improved the prognosis of luminal and HER2-positive cancers, chemotherapy remains the mainstay of 
treatment for TNBC. Furthermore, chemotherapy remains important in combination with targeted therapies in the metastatic setting. Current treatments, in particular chemotherapy, have several limitations, including long-term toxic effects, lack of specificity for cancer cells, and emergence of therapy resistance. Furthermore, the inherent genomic and molecular heterogeneity of breast tumors make cancer treatment particularly challenging. Thus, the development of novel strategies to increase the effectiveness of current therapy, reduce side effects, and reverse treatment resistance is an urgent clinical need.

The efficiency of combinatorial treatments is demonstrated by the approval of antibodyconjugated chemotherapeutics, such as trastuzumab emtansine (T-DM1), for the treatment of latestage HER2-positive breast cancer. In this context, the combination between standard anti-cancer treatments and gene therapy is emerging as a promising approach for the treatment of cancer. Besides their direct therapeutic application, monoclonal antibodies can be used to confer target selectivity to a wide range of nanoparticles [17]. For instance, trastuzumab functionalization of nanocomplexes enveloping DNA or RNA molecules, including siRNA against B-cell lymphomaextra large $(B C L-X L)$, CXC chemokine receptor 4 (CXCR4), HER2, PLK1, and signal transducer and activator of transcription-3 (STAT3), can be used for the specific targeting of HER2overexpressing breast cancer cells, resulting in an effective therapeutic strategy that combine targeted and gene therapies [27, 37, 47, 80, 81].

Even though several nanoparticles-based chemotherapeutic drugs, including liposomal doxorubicin, taxanes and cisplatin, are approved or are undergoing clinical trials for breast cancer, monoclonal antibodies can be used as a molecular tag to specifically deliver chemotherapeutics drugs into cancer cells $[16,17,32,82]$. A potential limitation of this latter approach, especially for TNBC, is represented by the lack of cell-specific markers beyond estrogen receptor (ER) and HER2 in breast cancer. Importantly, combination treatments of conventional chemotherapy and antisense oligonucleotides targeting genes responsible of multi-drug resistance (e.g. MDRI) or cancer stem cells (e.g. ALDH1, CD44) have been evaluated in breast cancer [18, 19, 83]. 


\subsection{Therapeutic stem cells}

Human MSCs are self-renewing multipotent cells. These stem cells properties, together with the tumor-homing ability, immunomodulatory functions, and low immunogenicity made stem cellbased systems an attractive approach for cancer therapy. However, one of the most interesting approaches is the use of MSCs as novel delivery vehicles for anti-cancer drugs or genetic materials to primary or metastatic cancers. In particular, MSCs have been used for suicide gene therapy, involving the introduction into tumor cells of a transgene that induce cancer cell death [84]. For instance, the use of MSCs as vehicles for the delivery of tumor necrosis factor-related apoptosisinducing ligands (TRAIL) or oncolytic viruses expressing the sodium iodine symporter demonstrated significant anti-tumor effects in in vivo models of breast cancer [84].

It is worth noting that MSCs have a well-known role in tumor initiation, progression, and resistance to therapies [85]. Furthermore, MSCs have been also demonstrated to be involved in local feedback loops with the tumor immune microenvironment, supporting aggressiveness and metastatic dissemination in breast cancer [85]. These tumor-promoting functions have raised concerns about the potential clinical application of MSCs for gene therapy. Indeed, several strategies have been proposed to target the tumor MSC pools, including suicide gene therapy, and immunostimulatory approaches [85].

\subsection{Genome editing}

Over the past two decades, different genome engineering technologies have been extensively used for research purposes to understand the role of specific genes in cancer development, progression and therapeutic response [3]. Recent advancement in the genome editing systems has led to a significant increase of the efficiency and specificity of gene targeting using site-specific endonucleases, including ZFNs, TALENs, and CRIPR/Cas9 [2]. ZFPs and TALENs, which are composed of a customized DNA-binding module and a non-specific DNA cleavage domain, can 
generate multiple genetic modifications by inducing DNA double-strand breaks (DSBs) that stimulate the error-prone non-homologous end joining or the more specific homologous recombination pathways [3, 74]. Even though these genome engineering methods may partly overcome the limitations of RNAi techniques, their therapeutic application is restricted by the complexity of designing the custom endonucleases. More recently, there has been growing interest on the more versatile RNA-guided CRISPR/Cas9 system, which has the potential to revolutionize the field of genome engineering for personalized medicine by overcoming many of the limitations of other editing methods. Basically, the DNA endonuclease Cas9 can be specifically guided to a target site using a single-guide RNA (sgRNA), inducing DSBs that initiate the DNA repair processes [3]. Stable Cas9-mediated gene modification can be accomplished through the transient transfection of plasmid DNA encoding Cas9 endonuclease and sgRNA, or through the stable deliver of CRISPR components using viral vectors [3]. The efficiency of this system allows the rapid and powerful engineering of human stem and cancer cells, as well as components of the immune system in vivo and ex vivo for therapeutic purposes.

Gene expression profiles and novel sequencing-based technologies have increased our knowledge of genetic aberrations and deregulated oncogenic pathways, which, together with the host immune response, affect breast cancer development and progression [86]. Several recurrent mutations and a wide range of rare mutations in oncogenes and tumor suppressor genes, including BRCA1/2, GATA3, MAP3K1, MLL3, PIK3CA, PTEN, RB1, and TP53, have been found with different frequencies in breast cancer subtypes [86]. These loss-of-function or gain-of-function mutations can be targeted by the CRISPR/Cas9 system to restore the physiological function of each gene and downstream pathways. Furthermore, CRISPR/Cas9 has also the potential for epigenome and gene expression modulation through the specific targeting of deregulated miRNAs $[87,88]$.

In recent years, considerable research efforts have focused on the relevance of host immune anti-cancer response and on the opportunity for immunotherapy applications in breast cancer [89]. In particular, increasing attention has been paid to the blockade of inhibitory pathways, such as the 
programmed death 1 (PD-1)/PD-L1 signaling [89]. Beyond the development of specific anti-PD1/PD-L1 antibodies, the CRISPR/Cas9 system has been used to genetically modify the PD-1 locus in $\mathrm{T}$ cells, leading to increased $\mathrm{T}$ cell effector activity [88]. Furthermore, the genetic engineering of autologous tumor-specific $\mathrm{T}$ cells for adoptive $\mathrm{T}$ cell therapy can be efficiently achieved with the CRISPR/Cas9 system [88]. In this regard, genetic engineering allows the modification of T cell's specificity toward a patient's cancer by introducing a cloned T cell receptor (TCR), or a chimeric antigen receptor (CAR), as discussed in the next paragraph [90].

\subsection{Immunomodulatory therapies}

Beyond the growing interest in directly targeting the immune system through the blockade of inhibitory pathways, such as the PD-1/PD-L1 or the cytotoxic T-lymphocyte-associated antigen-4 (CTLA-4) pathways in breast cancer, the genetic engineering of autologous $\mathrm{T}$ lymphocytes or dendritic cells (DCs), and cancer vaccines represent powerful strategies to generate a specific antitumor immune response against a patient's cancer (Figure 2).

Using ex vivo adoptive gene transfer, $\mathrm{T}$ cells can be genetically modified to express a novel TCR or a CAR to specifically recognize a tumor-associated antigen. The TCR interacts with an antigenic peptide presented by the human leucocyte antigen (HLA) class I complex, and induces the T cell-mediated cytolysis of the target cell (Figure 2) [91]. Virtually, TCRs have the potential to target all cancer-associated antigens, including mutated polypeptides from endogenous proteins. Although considerable efforts have been directed towards the development of efficient TCR engineering strategies, the frequent down-regulation of HLA class I during tumor progression, the requirement compatibility between HLA haplotype of the patient and the TCR, and disappointing results from early clinical trials have reshaped research directions $[1,91]$. A CAR is a transmembrane molecule, which combines an extracellular tumor-specific fragment from a monoclonal antibody with intracellular signaling motifs capable of boosting $\mathrm{T}$ cell activation [91]. In contrast to the TCR, upon the binding to a tumor-associated antigen through its antibody moiety, 
the CAR induces apoptosis in the target cell independently of HLA, allowing the recognition of targets other than peptides, and a broader selection of patients eligible for adoptive therapy (Figure 2) [90]. However, CAR T cell-based therapy requires cancer-related targets to be cell surface antigens, thus excluding the recognition of all mutated intracellular proteins [91].

Another approach to potentiate anti-cancer immune response is the use of cancer vaccines, which provide the patient's immune system with specific tumor antigens. Therapeutic cancer vaccines can derive from whole tumor cells, protein antigens, peptides, DNA, or DCs (Figure 2) [92-94]. Whole-cell vaccines are able to induce an immune response against multiple tumor targets [94]. Clinical trials evaluating cell-based vaccines failed to show a consistent clinical benefit in cancer patients, although demonstrating the development of an immune response [94, 95]. However, different strategies other than the administration of intact cancer cells are currently being explored, including genetic engineering of tumor cells and fusion with DCs [93, 94]. For instance, autologous whole tumor cells have been modified to express granulocyte-macrophage colonystimulating factor (GM-CSF) and to downregulate transforming growth factor (TGF)- $\beta$ to enhance the recruitment of DCs and boost the activation of anti-tumor immune response [95].

As any effective anti-tumor immune response involves the processing and presentation of cancer-related antigens by DCs, these cells have been proposed to be crucial for the development of an efficient vaccination strategy. Beyond the use of viral vectors, DCs have been explored as a delivery vehicle with inherent adjuvant properties (Figure 2). Indeed, DCs derived from circulating blood precursors can be loaded with tumor antigens or peptides, transfected with tumor-derived RNA or DNA, or genetically engineered to produce a specific tumor antigen, and then infused back to the patient, thus serving as a delivery vehicle with inherent adjuvant properties $[93,94,96]$. This approach has demonstrated clinical efficacy in certain types of cancer, leading to the approval of a DC-based vaccine for the treatment of metastatic hormone-refractory prostate cancer [96]. However, there are challenges related to the use of DC-based vaccine, including the complexity of ex vivo manipulation, and the existence of multiple DCs subsets with different ability to process and 
present antigens and to modulate the activation of T cells $[94,96]$. Although several strategies are being evaluated to improve the effectiveness of DC-based vaccines, including the appropriate selection of DCs subset and adjuvants, and the enhancement of DCs functionalities, their clinical utility is still unclear $[94,96]$.

Finally, active immunotherapies can also take advantage from the use of protein antigens, peptides and nucleic acids from tumor cells (Figure 2) [92-94]. Several strategies for the delivery of genes encoding tumor antigens able to elicit a specific immune response have been described [92]. Advancement for the implementation of delivery systems, development of adjuvants and other immune stimulatory signals, have led to the evaluation of DNA-based vaccines in clinical trials for different diseases, including breast cancer [92]. In addition, peptides derived from tumor-associated antigens, most of which are based on wild-type protein sequences overexpressed by cancer cells, can directly bind to HLA molecules, thus overcoming the need for antigen processing and presentation, and allowing an efficient activation of $\mathrm{T}$ cells [94]. Despite these promising assumptions, single peptides demonstrated limited efficacy in clinical studies, and the addition of enhancers of immune response (e.g. IL-2) are being investigated [94].

A basic concept to highlight is that the activity and therapeutic efficiency of all immunebased gene therapies are dependent on the recognition of a specific tumor target. Ideally, the selected antigen would be non-essential to normal human tissues and expressed only by cancer cells. However, the identification of selective cancer antigens is challenging. Accordingly, therapies with CAR and engineered TCR are often associated with significant toxicities due to the recognition of the antigen expressed on normal tissues [90]. In this regard, neoantigens may represent valuable targets, as they are exclusive to tumor cells and arise from recurrent driver mutations that are crucial for the malignant phenotype [90].

\section{Gene therapies under clinical evaluation for breast cancer}


The majority of clinical trials in gene therapy (64.4\%) have been undertaken in cancer patients [97]. A broad spectrum of delivery systems, including viral vectors, inorganic nanoparticles, and lipid- or polymeric-based nanovectors has been investigated. Furthermore, a wide range of strategies, including replacement gene therapy, oncolytic virotherapy, suicide gene therapy, RNAi, and immunotherapy has been evaluated. Even though several major challenges must still be addressed, clinical trials of gene therapy are beginning to show an improved safety profile and substantial therapeutic benefits. A growing number of clinical trials of gene therapy and immune-based therapy are being conducted for the treatment of breast cancer (Table 2).

The concerns about the safety of virus-based approaches led to a substantial decline of their application in clinical trials [97]. However, two clinical trials of TP53-targeting therapy in combination with chemotherapy, and a phase $1 / 2$ study of Rexin-G, consisting of a viral envelope carrying cyclin G1 gene, have been recently completed in advanced or metastatic breast cancer, and their results are awaited (Table 2). Moreover, talimogene laherparepvec and JX-594 oncolytic viruses, and the retroviral vector Toca 511 for suicide gene therapy, are now being evaluated for triple negative and advanced/metastatic breast cancer (Table 2).

A growing interest is now focused on immunomodulatory therapies for breast cancer (Table 2). This is reflected by the evaluation of two anti-HER2 vaccines - NeuVax and AE37 - in phase 23 studies for the treatment of HER2-positive breast cancer (Table 2). Viral vectors are also emerging as important delivery systems for immunotherapy in breast cancer. Recent results from a randomized, phase 2 clinical trial suggested that the combination of a recombinant vaccinia virus producing the carcinoembryonic antigen (CEA) and mucin-1 (MUC-1), together with the triad of costimulatory molecules B7.1, ICAM-1 and LFA-3 (TRICOM), with docetaxel might provide a clinical benefit in metastatic breast cancer [98]. Viral vectors encoding a wide range of proteins, including TP53, CEA, IL-12, HER2, the EMT driver brachyury, and a fusion protein of human MUC-1 and CD40 ligand, are currently under clinical investigation for the boosting of anti-tumor immune response (Table 2). Furthermore, plasmid DNA has also been employed for cancer vaccine 
and immunotherapy. For instance, plasmids DNA encoding CYP1B1, IL-12, mammaglobin-A, PRAME and PSMA, are in early clinical evaluation for breast cancer treatment (Table 2). Engineered TCRs and CARs are also being evaluated in clinical trials for their ability to "educate" the host immune systems to recognize specific tumor antigens, including CD133, CEA, EpCAM, HER2, mesothelin, MET proto-oncogene, NY-ESO-1, ROR1, and TP53 (Table 2). Furthermore, DCs-based vaccination strategies are under clinical evaluation for the treatment of breast cancer (Table 2). All these strategies are often combined with the administration of immunostimulatory cytokines, including IL-2, GM-CSF, and granulocyte colony-stimulating factor (G-CSF), to boost their anti-tumor activity (Figure 2).

Overall, siRNA- and miRNA-based therapies have not yet entered the clinic, with only one study evaluating RNAi technology in breast cancer (Table 2).

\section{Expert opinion}

Gene therapy has certainly the potential to impact clinical research. However, major limitations must still be overcome. In particular, the safety and efficiency of delivery systems should be improved by further engineering their composition, immunogenicity, and functionalization, and by developing novel strategies to potentiate their specificity toward cancer cells. Furthermore, the current efficiency of these systems may be sub-therapeutic for certain diseases, and off-target genomic alterations may cause detrimental effects. Thus, the rate and specificity of gene modification must be increased before considering gene therapy as a concrete option for the treatment of cancer patients. Beyond virus-based approaches, nanoparticles have several advantages, including the capability to carry a superior and more varied drug cargo, and the opportunity of being differently functionalized. These versatile systems have thus a great potential to be further engineered and to be used for combination treatment between conventional anti-cancer drugs and gene-based therapy. MCSs deserve additional consideration as novel delivery vectors, due to their tumor-homing ability and low immunogenicity. However, the tumor-promoting 
functions of MSCs, as well as their involvement in therapy resistance, must be taken into account, especially in several types of cancer, such as a subgroup of TNBC. In particular, the relationship between MSCs functions and the tumor immune microenvironment is emerging as a crucial factor driving tumor development, progression and therapy resistance. Thus, additional studies are required to understand the role of MSCs in cancer tissues, and to identify any oncogenic change that can potentially occur following their engraftment at the tumor site.

Even though gene therapy was classically envisioned as an option to treat a disease at a genetic level, further efforts are also required to boost the development of RNAi technologies, and to increase the number of siRNA- and miRNA-based therapeutics being tested in clinical studies, ultimately translating this knowledge into clinical practice. It is also worth noting that each cancer is a unique genomic entity, which can carry different types of mutations on the same gene, and a combination of multiple distinct mutated genes and noncoding regions. Such a complex landscape can differentially impair multiple cancer-related pathways. In this context, advancement in genome editing technologies, such as CRISPR/Cas9 system, can be used to specifically modify multiple different sites simultaneously, with the potential to reshape research directions and improve the clinical management of cancer patients. However, several technical challenges must still be addressed, and the functional relevance of each mutations, as well as their overall effects on signaling pathways should be deeply dissect before considering genome editing techniques as potential therapeutic strategy in humans.

Besides conventional gene therapy approaches, immune-based approaches have received increasing attention as new therapeutic options for patients with breast cancer, especially HER2positive and TNBC. Immunomodulation of cancer cells and the tumor microenvironment trough the selective delivery of cytokines and chemokines, as well as the development of cancer vaccine to boost anti-tumor immunity have been evaluated in preclinical and clinical studies of breast cancer, proving evidence of immune activation. However, substantial evidence of clinical activity is still lacking. Therapeutic cancer vaccines and modified TCRs and CARs have proven to be challenging 
irrespective to the engineering strategy employed, the type of antigen used, and the supportive cytokine regimen provided. Noteworthy, several types of cancer, including breast cancer, have not been traditionally considered an immunogenic tumor. In breast cancer, there is a paucity of both antigens specific of cancer cells, and neoantigens due to the reduced number of recurrent mutations in cancer-related genes. Expanding the use of immune-based therapies to treat cancer patients will require major technical improvements, the identification of antigen specific of cancer cells, and further knowledge on the biological mechanisms underlying immune editing, antigen processing and presentation, and long-term effects of anti-cancer immune response.

\section{Article highlights box}

- Gene therapy has a great potential to improve the clinical management of breast cancer patients.

- Further efforts are required to increase the clinical application of RNAi-based therapeutics.

- Genome editing and stem cell-based systems, may certainly contribute to translate gene therapy into clinical practice.

- Immune-based strategies is emerging as an attractive therapeutic opportunity for specific breast cancer patients.

- Technical challenges need to be addressed before using gene therapy as a concrete option for the treatment of breast cancer. 
List of abbreviations: CRISPR/Cas9: clustered regularly interspaced short palindromic repeat /associated protein 9; DCs: Dendritic cells; EMT: epithelial-to-mesenchymal transition; G-CSF: Granulocyte colony-stimulating factor; GM-CSF: Granulocyte-macrophage colony-stimulating factor; HER2: erb-b2 receptor tyrosine kinase 2; HSV-1: herpes simplex virus type 1; IL-2: Interleukin-2; MAPK: mitogen-activated protein kinase; MDR1: Multidrug resistance gene 1; miRNA: microRNA; MSCs: mesenchymal stem cells; PEG: polyethylene glycol; PEI: polyethylenimines; PI3K: phosphatidylinositol-4,5-bisphosphate 3-kinase; RNAi: RNA interference; siRNA: small-interfering RNA; TALEN: transcription activator-like effector nuclease; TCR: T cell receptor; TNBC: Triple negative breast cancer; ZFN: zinc-finger nuclease;

\section{Acknowledgements}

The authors are grateful to Associazione Italiana Ricerca sul Cancro for support to L.S. (AIRC Grant 6251); Fondazione Italiana Ricerca sul Cancro for support to G.B. (FIRC fellowship 18328); Fondazione Regionale per la Ricerca Biomedica for support to F.C. and M.T.

\section{Declaration of interest}

The authors state no conflict of interest and have received no payment for preparation of this manuscript. The authors confirm that the submitted figures and tables are original and have not previously been published. 


\section{References}

1. Naldini L. Gene therapy returns to centre stage. Nature 2015;526:351-60.

2. Yin H, Kanasty RL, Eltoukhy AA, et al. Non-viral vectors for gene-based therapy. Nat Rev Genet 2014;15:541-55.

3. Sanchez-Rivera FJ, Jacks T. Applications of the CRISPR-Cas9 system in cancer biology. Nat Rev Cancer 2015; 15:387-95.

4. Chira S, Jackson CS, Oprea I, et al. Progresses towards safe and efficient gene therapy vectors. Oncotarget 2015;6:30675-703.

5. McCrudden CM, McCarthy HO. Current status of gene therapy for breast cancer: progress and challenges. Appl Clin Genet 2014;7:209-20.

6. Amer MH. Gene therapy for cancer: present status and future perspective. Mol Cell Ther 2014;2:27.

7. Kumar SR, Markusic DM, Biswas M, et al. Clinical development of gene therapy: results and lessons from recent successes. Mol Ther Methods Clin Dev 2016;3:16034.

8. Gandhi NS, Tekade RK, Chougule MB. Nanocarrier mediated delivery of siRNA/miRNA in combination with chemotherapeutic agents for cancer therapy: current progress and advances. J Control Release 2014;194:238-56.

9. Nastiuk KL, Krolewski JJ. Opportunities and challenges in combination gene cancer therapy. Adv Drug Deliv Rev 2016;98:35-40.

10. Swartz MA, Iida N, Roberts EW, et al. Tumor microenvironment complexity: emerging roles in cancer therapy. Cancer Res 2012;72:2473-80.

11. Kotterman MA, Chalberg TW, Schaffer DV. Viral Vectors for Gene Therapy: Translational and Clinical Outlook. Annu Rev Biomed Eng 2015;17:63-89.

12. Miest TS, Cattaneo R. New viruses for cancer therapy: meeting clinical needs. Nat Rev Microbiol 2014;12:23-34. 
13. Lichty BD, Breitbach CJ, Stojdl DF, et al. Going viral with cancer immunotherapy. Nat Rev Cancer 2014;14:559-67.

14. Pahle J, Walther W. Vectors and strategies for nonviral cancer gene therapy. Expert Opin Biol Ther 2016;16:443-61.

15. Truffi M, Sorrentino L, Mazzucchelli S, et al. What Is the Role of Nanotechnology in Diagnosis and Treatment of Metastatic Breast Cancer? Promising Scenarios for the Near Future. Journal of Nanomaterials 2016;2016:16.

16. Cho K, Wang X, Nie S, et al. Therapeutic nanoparticles for drug delivery in cancer. Clin Cancer Res 2008;14:1310-6.

17. Vago R, Collico V, Zuppone S, et al. Nanoparticle-mediated delivery of suicide genes in cancer therapy. Pharmacol Res 2016;111:619-41.

18. Stoff-Khalili MA, Dall P, Curiel DT. Gene therapy for carcinoma of the breast. Cancer Gene Ther 2006;13:633-47.

19. Dong D, Gao W, Liu Y, et al. Therapeutic potential of targeted multifunctional nanocomplex co-delivery of siRNA and low-dose doxorubicin in breast cancer. Cancer Lett 2015;359:178-86.

20. Shen H, Mittal V, Ferrari M, et al. Delivery of gene silencing agents for breast cancer therapy. Breast Cancer Res 2013;15:205.

21. Prabhakar U, Maeda H, Jain RK, et al. Challenges and key considerations of the enhanced permeability and retention effect for nanomedicine drug delivery in oncology. Cancer Res $2013 ; 73: 2412-7$.

22. Ozpolat B, Sood AK, Lopez-Berestein G. Liposomal siRNA nanocarriers for cancer therapy. Adv Drug Deliv Rev 2014;66:110-6.

23. Li Y, Lee RJ, Yu K, et al. Delivery of siRNA using lipid nanoparticles modified with cell penetrating peptide. ACS Appl Mater Interfaces 2016;8:26613-21. 
24. Gujrati M, Vaidya AM, Mack M, et al. Targeted dual pH-sensitive lipid ECO/siRNA selfassembly nanoparticles facilitate in vivo cytosolic sieIF4E delivery and overcome paclitaxel resistance in breast cancer therapy. Adv Healthc Mater 2016. doi: 10.1002/adhm.201600677.

25. Parvani JG, Gujrati MD, Mack MA, et al. Silencing beta3 integrin by targeted ECO/siRNA nanoparticles inhibits EMT and metastasis of triple-negative breast cancer. Cancer Res 2015;75:2316-25.

- This study demonstrates that the administration of $\beta 3$ integrin siRNA via lipid ECO-based nanoparticles effectively silences $\beta 3$ integrin expression, attenuates TGF $\beta$-mediated epithelial-tomesenchymal transition, and reduces metastases and relapse in a triple-negative breast cancer mouse model.

26. Zuckerman JE, Davis ME. Clinical experiences with systemically administered siRNAbased therapeutics in cancer. Nat Rev Drug Discov 2015;14:843-56.

27. Yao YD, Sun TM, Huang SY, et al. Targeted delivery of PLK1-siRNA by ScFv suppresses Her2+ breast cancer growth and metastasis. Sci Trans1 Med 2012;4:130ra48.

- This report shows that the delivery of a PLK1-siRNAs complexed with a HER2 single-chain variable fragments-protamine fusion protein suppress proliferation and induced apoptosis in HER2positive breast cancer cell lines and breast cancer mice models.

28. Tang Z, He C, Tian H, et al. Polymeric nanostructured materials for biomedical applications. Progress in Polymer Science 2016;60:86-128.

29. Bolcato-Bellemin AL, Bonnet ME, Creusat G, et al. Sticky overhangs enhance siRNAmediated gene silencing. Proc Natl Acad Sci U S A 2007;104:16050-5.

30. Talluri SV, Kuppusamy G, Karri VV, et al. Lipid-based nanocarriers for breast cancer treatment - comprehensive review. Drug Deliv 2016;23:1291-305.

31. Zhou H, Wei J, Dai Q, et al. $\mathrm{CaCO}_{3} / \mathrm{CaIP}_{6}$ composite nanoparticles effectively deliver AKT1 small interfering RNA to inhibit human breast cancer growth. Int $\mathrm{J}$ Nanomedicine 2015;10:4255-66. 
32. Lee J, Chatterjee DK, Lee MH, et al. Gold nanoparticles in breast cancer treatment: promise and potential pitfalls. Cancer Lett 2014;347:46-53.

33. Zhou Z, Kennell C, Lee JY, et al. Calcium phosphate-polymer hybrid nanoparticles for enhanced triple negative breast cancer treatment via co-delivery of paclitaxel and miR-221/222 inhibitors. Nanomedicine 2016. doi: 10.1016/j.nano.2016.07.016.

34. Li C, Li L, Keates AC. Targeting cancer gene therapy with magnetic nanoparticles. Oncotarget 2012;3:365-70.

35. Ekin A, Karatas OF, Culha M, et al. Designing a gold nanoparticle-based nanocarrier for microRNA transfection into the prostate and breast cancer cells. J Gene Med 2014;16:331-5.

36. Shu D, Li H, Shu Y, et al. Systemic delivery of anti-miRNA for suppression of triple negative breast cancer utilizing RNA nanotechnology. ACS Nano 2015;9:9731-40.

37. Guo P. The emerging field of RNA nanotechnology. Nat Nanotechnol 2010;5:833-42.

38. Lee H, Lytton-Jean AK, Chen Y, et al. Molecularly self-assembled nucleic acid nanoparticles for targeted in vivo siRNA delivery. Nat Nanotechnol 2012;7:389-93.

- In this study, self-assembled DNA tetrahedral nanoparticles are able to efficiently deliver siRNAs into cells and silence target genes in tumors, by precisely controlling of spatial orientation and density of cancer-targeting ligands on the nanoparticle surface.

39. Cristofanilli M, Krishnamurthy S, Guerra L, et al. A nonreplicating adenoviral vector that contains the wild-type p53 transgene combined with chemotherapy for primary breast cancer: safety, efficacy, and biologic activity of a novel gene-therapy approach. Cancer 2006;107:935-44.

40. Wang K, Huang Q, Qiu F, et al. Non-viral Delivery Systems for the Application in p53 Cancer Gene Therapy. Curr Med Chem 2015;22:4118-36

41. Vidal SJ, Rodriguez-Bravo V, Galsky M, et al. Targeting cancer stem cells to suppress acquired chemotherapy resistance. Oncogene 2014;33:4451-63. 
42. Hayward SL, Francis DM, Kholmatov P, et al. Targeted Delivery of MicroRNA125a-5p by engineered lipid nanoparticles for the treatment of HER2 positive metastatic breast cancer. J Biomed Nanotechnol 2016;12:554-68.

43. Yu MZ, Pang WH, Yang T, et al. Systemic delivery of siRNA by T7 peptide modified coreshell nanoparticles for targeted therapy of breast cancer. Eur J Pharm Sci 2016;92:39-48.

44. Devulapally R, Sekar NM, Sekar TV, et al. Polymer nanoparticles mediated codelivery of antimiR-10b and antimiR-21 for achieving triple negative breast cancer therapy. ACS Nano 2015;9:2290-302.

45. Elazar V, Adwan H, Bauerle T, et al. Sustained delivery and efficacy of polymeric nanoparticles containing osteopontin and bone sialoprotein antisenses in rats with breast cancer bone metastasis. Int J Cancer 2010;126:1749-60.

46. Devulapally R, Sekar TV, Paulmurugan R. Formulation of anti-miR-21 and 4hydroxytamoxifen co-loaded biodegradable polymer nanoparticles and their antiproliferative effect on breast cancer cells. Mol Pharm 2015;12:2080-92.

47. Palanca-Wessels MC, Booth GC, Convertine AJ, et al. Antibody targeting facilitates effective intratumoral siRNA nanoparticle delivery to HER2-overexpressing cancer cells. Oncotarget 2016;7:9561-75.

- This paper demonstrates that antibodies directed against highly expressed cancer cell surface receptors (HER2) linked to a polymeric nanocarrier can be used to generate a tumor-specific recognition.

48. Wu Y, Zhang Y, Zhang W, et al. Reversing of multidrug resistance breast cancer by codelivery of P-gp siRNA and doxorubicin via folic acid-modified core-shell nanomicelles. Colloids Surf B Biointerfaces 2016;138:60-9.

49. Ren X, Liu L, Zhou Y, et al. Nanoparticle siRNA against BMI-1 with a PolyethylenimineLaminarin Conjugate for Gene Therapy in Human Breast Cancer. Bioconjug Chem 2016;27:66-73. 
50. Bai M, Shen M, Teng Y, et al. Enhanced therapeutic effect of adriamycin on multidrug resistant breast cancer by the ABCG2-siRNA loaded polymeric nanoparticles assisted with ultrasound. Oncotarget 2015;6:43779-90.

51. Finlay J, Roberts CM, Lowe G, et al. RNA-based TWIST1 inhibition via dendrimer complex to reduce breast cancer cell metastasis. Biomed Res Int 2015;2015:382745.

52. Yang H, Deng L, Li T, et al. Multifunctional PLGA Nanobubbles as Theranostic Agents: Combining Doxorubicin and P-gp siRNA Co-delivery into human breast cancer cells and ultrasound cellular imaging. J Biomed Nanotechnol 2015;11:2124-36.

53. Tekie FS, Atyabi F, Soleimani M, et al. Chitosan polyplex nanoparticle vector for miR-145 expression in MCF-7: Optimization by design of experiment. Int J Biol Macromol 2015;81:828-37.

54. Yin T, Wang L, Yin L, et al. Co-delivery of hydrophobic paclitaxel and hydrophilic AURKA specific siRNA by redox-sensitive micelles for effective treatment of breast cancer. Biomaterials 2015;61:10-25.

- This study reports a novel redox-sensitive micellar system developed for tumor-targeted codelivery of paclitaxel and AURKA-siRNA, substantially increasing the anti-tumor efficacy of chemotherapy.

55. Kaushal N, Durmaz YY, Bao L, et al. "Smart" Nanoparticles Enhance the Cytoplasmic Delivery of Anti-RhoC Silencing RNA and Inhibit the Migration and Invasion of Aggressive Breast Cancer Cells. Mol Pharm 2015;12:2406-17.

56. Tang S, Yin Q, Su J, et al. Inhibition of metastasis and growth of breast cancer by $\mathrm{pH}-$ sensitive poly (beta-amino ester) nanoparticles co-delivering two siRNA and paclitaxel. Biomaterials 2015;48:1-15.

57. Yhee JY, Song S, Lee SJ, et al. Cancer-targeted MDR-1 siRNA delivery using self-crosslinked glycol chitosan nanoparticles to overcome drug resistance. J Control Release 2015;198:1-9. 
- In this study, MDR1 targeting siRNAs is efficiently delivered to chemo-resistant mammary tumors using a glycol chitosan nanoparticles, which demonstrate stability, siRNAs protection from enzymatic degradation, and inhibition of tumor growth without systemic toxicity.

58. Qian J, Xu M, Suo A, et al. Folate-decorated hydrophilic three-arm star-block terpolymer as a novel nanovehicle for targeted co-delivery of doxorubicin and Bcl-2 siRNA in breast cancer therapy. Acta Biomater 2015;15:102-16.

59. Tangudu NK, Verma VK, Clemons TD, et al. RNA interference using c-myc-conjugated nanoparticles suppresses breast and colorectal cancer models. Mol Cancer Ther 2015;14:1259-69.

60. Alinejad V, Hossein Somi M, Baradaran B, et al. Co-delivery of IL17RB siRNA and doxorubicin by chitosan-based nanoparticles for enhanced anticancer efficacy in breast cancer cells. Biomed Pharmacother 2016;83:229-40.

61. Jadidi-Niaragh F, Atyabi F, Rastegari A, et al. Downregulation of CD73 in 4T1 breast cancer cells through siRNA-loaded chitosan-lactate nanoparticles. Tumour Biol 2016;37:8403-12.

62. Yoo B, Kavishwar A, Ross A, et al. Combining miR-10b-targeted nanotherapy with lowdose doxorubicin elicits durable regressions of metastatic breast cancer. Cancer Res 2015;75:440715.

- In this report, the combination of a miR-10b-inhibitory nanodrug with low-dose anthracycline exerts an anti-proliferative and pro-apoptotic effect, achieving a complete regressions of metastatic disease in a mouse model of metastatic breast cancer.

63. Hardie J, Jiang Y, Tetrault ER, et al. Simultaneous cytosolic delivery of a chemotherapeutic and siRNA using nanoparticle-stabilized nanocapsules. Nanotechnology 2016;27:374001.

64. Rajput S, Puvvada N, Kumar BN, et al. Overcoming Akt induced therapeutic resistance in breast cancer through sirna and thymoquinone encapsulated multilamellar gold niosomes. Mol Pharm 2015;12:4214-25.

65. Khaled SZ, Cevenini A, Yazdi IK, et al. One-pot synthesis of pH-responsive hybrid nanogel particles for the intracellular delivery of small interfering RNA. Biomaterials 2016;87:57-68. 
- This report describes a novel method to synthetize hybrid nanoparticles formed by an inorganic silica core and an organic pH-responsive hydrogel shell that can be loaded with CXCR4siRNAs, accumulating at tumor site and decreasing CXCR4 expression in a mouse model of human breast cancer.

66. Liu L, Li H, Zhang M, et al. Effects of targeted nano-delivery systems combined with hTERT-siRNA and Bmi-1-siRNA on MCF-7 cells. Int J Clin Exp Pathol 2015;8:6674-82.

67. $\mathrm{Gu} \mathrm{S}, \mathrm{Hu} \mathrm{Z}$, Ngamcherdtrakul W, et al. Therapeutic siRNA for drug-resistant HER2positive breast cancer. Oncotarget 2016;7:14727-41.

68. Li T, Shen $\mathrm{X}$, Chen $\mathrm{Y}$, et al. Polyetherimide-grafted $\mathrm{Fe}_{3} \mathrm{O}_{4} @ \mathrm{SiO}_{2}$ nanoparticles as theranostic agents for simultaneous VEGF siRNA delivery and magnetic resonance cell imaging. Int J Nanomedicine 2015;10:4279-91.

69. Li M, Li Y, Huang X, et al. Captopril-polyethyleneimine conjugate modified gold nanoparticles for co-delivery of drug and gene in anti-angiogenesis breast cancer therapy. J Biomater Sci Polym Ed 2015;26:813-27.

70. Arami S, Mahdavi M, Rashidi MR, et al. Novel polyacrylate-based cationic nanoparticles for survivin siRNA delivery combined with mitoxantrone for treatment of breast cancer. Biologicals 2016. doi: 10.1016/j.biologicals.2016.09.005.

71. Conde J, Oliva N, Atilano M, et al. Self-assembled RNA-triple-helix hydrogel scaffold for microRNA modulation in the tumour microenvironment. Nat Mater 2016;15:353-63.

- This report demonstrates that a self-assembled RNA-triple-helix nanoparticle including a miRNA mimic and an antagomiRNA is able to synergistically abrogate tumors, and to modulate the expression of endogenous miRNAs in a triple-negative breast cancer mouse model.

72. Subramanian N, Kanwar JR, Kanwar RK, et al. Targeting Cancer Cells Using LNAModified Aptamer-siRNA Chimeras. Nucleic Acid Ther 2015;25:317-22.

73. Lin S, Gregory RI. MicroRNA biogenesis pathways in cancer. Nat Rev Cancer $2015 ; 15: 321-33$. 
74. Bottai G, Pasculli B, Calin GA, et al. Targeting the microRNA-regulating DNA damage/repair pathways in cancer. Expert Opin Biol Ther 2014;14:1667-83.

75. De Mattos-Arruda L, Bottai G, Nuciforo PG, et al. MicroRNA-21 links epithelial-tomesenchymal transition and inflammatory signals to confer resistance to neoadjuvant trastuzumab and chemotherapy in HER2-positive breast cancer patients. Oncotarget 2015;6:37269-80.

- In this paper, the expression of tissue miR-21 is demonstrated to be associated with residual disease in HER2-positive breast cancer patients, and affect the response to both trastuzumab and chemotherapy triggering an inflammatory pathway, suggesting that using miR-21 could be used to revert trastuzumab and chemotherapy resistance in breast cancer.

76. Kleivi Sahlberg K, Bottai G, Naume B, et al. A serum microRNA signature predicts tumor relapse and survival in triple-negative breast cancer patients. Clin Cancer Res 2015;21:1207-14.

- In this study, a circulating miRNA signature is demonstrated to serve as a minimally invasive predictor of tumor relapse and overall survival for patients with triple negative breast cancer, indicating the relevance of miRNAs deregulation in breast cancer.

77. Jung EJ, Santarpia L, Kim J, et al. Plasma microRNA 210 levels correlate with sensitivity to trastuzumab and tumor presence in breast cancer patients. Cancer 2012;118:2603-14.

78. Paladini L, Fabris L, Bottai G, et al. Targeting microRNAs as key modulators of tumor immune response. J Exp Clin Cancer Res 2016;35:103.

79. Senkus E, Kyriakides S, Ohno S, et al. Primary breast cancer: ESMO Clinical Practice Guidelines for diagnosis, treatment and follow-up. Ann Oncol 2015;26 Suppl 5:v8-30.

80. Mann K, Kullberg M. Trastuzumab-targeted gene delivery to Her2-overexpressing breast cancer cells. Cancer Gene Ther 2016;23:221-8.

81. Jiang K, Li J, Yin J, et al. Targeted delivery of CXCR4-siRNA by scFv for HER2(+) breast cancer therapy. Biomaterials 2015;59:77-87.

- This article describe an approach to increase the specificity of CXCR4 siRNA delivery by using an anti-HER2 single-chain variable fragments antibody fused with an arginine nonamer peptide. 
This strategy is able to suppress tumor growth, reduce metastasis and prolong survival in HER2positive in vivo models, without inducing systemic toxicity and host immune response.

82. Cheng Z, Al Zaki A, Hui JZ, et al. Multifunctional nanoparticles: cost versus benefit of adding targeting and imaging capabilities. Science 2012;338:903-10.

83. Li SY, Sun R, Wang HX, et al. Combination therapy with epigenetic-targeted and chemotherapeutic drugs delivered by nanoparticles to enhance the chemotherapy response and overcome resistance by breast cancer stem cells. J Control Release 2015;205:7-14.

- This study demonstrates that nanoparticles loaded with chemotherapeutic drugs reduce the proportion of cancer stem cells and induce apoptosis, thus inhibiting breast cancer growth and enhancing chemotherapy sensitivity.

84. Dwyer RM, Khan S, Barry FP, et al. Advances in mesenchymal stem cell-mediated gene therapy for cancer. Stem Cell Res Ther 2010;1:25.

85. Pattabiraman DR, Weinberg RA. Tackling the cancer stem cells - what challenges do they pose? Nat Rev Drug Discov 2014;13:497-512.

86. Santarpia L, Bottai G, Kelly CM, et al. Deciphering and Targeting Oncogenic Mutations and Pathways in Breast Cancer. Oncologist 2016;21:1063-78.

87. Khan FA, Pandupuspitasari NS, Chun-Jie H, et al. CRISPR/Cas9 therapeutics: a cure for cancer and other genetic diseases. Oncotarget 2016. doi: 10.18632/oncotarget.9646.

88. Yi L, Li J. CRISPR-Cas9 therapeutics in cancer: promising strategies and present challenges. Biochim Biophys Acta 2016;1866:197-207.

89. Savas P, Salgado R, Denkert C, et al. Clinical relevance of host immunity in breast cancer: from TILs to the clinic. Nat Rev Clin Oncol 2016;13:228-41.

90. Klebanoff CA, Rosenberg SA, Restifo NP. Prospects for gene-engineered $\mathrm{T}$ cell immunotherapy for solid cancers. Nat Med 2016;22:26-36.

91. Essand M, Loskog AS. Genetically engineered T cells for the treatment of cancer. J Intern Med 2013;273:166-81. 
92. Tiptiri-Kourpeti A, Spyridopoulou K, Pappa A, et al. DNA vaccines to attack cancer: Strategies for improving immunogenicity and efficacy. Pharmacol Ther 2016;165:32-49.

93. Melero I, Gaudernack G, Gerritsen W, et al. Therapeutic vaccines for cancer: an overview of clinical trials. Nat Rev Clin Oncol 2014;11:509-24.

94. Makkouk A, Weiner GJ. Cancer immunotherapy and breaking immune tolerance: new approaches to an old challenge. Cancer Res 2015;75:5-10.

95. Chiang CL, Coukos G, Kandalaft LE. Whole Tumor Antigen Vaccines: Where Are We? Vaccines (Basel) 2015;3:344-72.

96. Palucka K, Banchereau J. Dendritic-cell-based therapeutic cancer vaccines. Immunity 2013;39:38-48.

97. Ginn SL, Alexander IE, Edelstein ML, et al. Gene therapy clinical trials worldwide to 2012 an update. J Gene Med 2013;15:65-77.

98. Heery CR, Ibrahim NK, Arlen PM, et al. Docetaxel Alone or in Combination With a Therapeutic Cancer Vaccine (PANVAC) in Patients With Metastatic Breast Cancer: A Randomized Clinical Trial. JAMA Oncol 2015;1:1087-95.

$\bullet \bullet$ In this randomized clinical trial, the combination of docetaxel and a virus-based cancer vaccine is demonstrated to improve clinical outcomes in patients with metastatic breast cancer. 
Table 1. Recent preclinical advances in nanotechnology for RNA-based therapy in breast cancer

\begin{tabular}{|c|c|c|c|c|c|}
\hline $\begin{array}{l}\text { Nanoparticle } \\
\text { type }\end{array}$ & Therapeutic agent & Target gene & Therapeutic effect & Nanocompound & Reference \\
\hline \multirow{3}{*}{$\begin{array}{l}\text { Lipid } \\
\text { nanoparticles }\end{array}$} & eIF4E-siRNA & $\begin{array}{l}\text { Eukaryotic translation } \\
\text { initiation factor } 4 \mathrm{E}\end{array}$ & $\begin{array}{l}\text { Resensitization of triple negative breast cancer to } \\
\text { paclitaxel }\end{array}$ & $\begin{array}{l}\text { Dual pH-sensitive RGD-PEG(HZ)- } \\
\text { ECO/sieIF4E }\end{array}$ & {$[24]$} \\
\hline & $\operatorname{miR}-125 \mathrm{a}$ & $\begin{array}{l}\text { HER2 proto-oncogene } \\
\text { and other miR-125a } \\
\text { targets }\end{array}$ & $\begin{array}{l}\text { Suppression of PI3K/AKT and MAPK hyperactivated } \\
\text { signaling pathways, cellular proliferation and migration } \\
\text { potential of HER2-positive metastatic breast cancer cells }\end{array}$ & HA-coated lipid nanoparticle platform & {$[42]$} \\
\hline & $\beta 3$ integrin-siRNA & $\beta 3$ integrin & $\begin{array}{l}\text { Attenuation of EMT and invasion; inhibition of 3-D } \\
\text { organoid growth; metastasis inhibition in triple negative } \\
\text { breast cancer in vivo }\end{array}$ & $\begin{array}{l}\text { RGD-targeted lipid-ECO-based nanoparticles } \\
(\mathrm{ECO} / \mathrm{si} \beta 3)\end{array}$ & {$[25]$} \\
\hline \multirow[t]{5}{*}{ Liposomes } & EGFR-siRNA & EGFR & $\begin{array}{l}\text { Inhibition of MCF-7 breast tumor growth in vitro and in } \\
\text { vivo }\end{array}$ & $\begin{array}{l}\text { siEGFR/protamine/CS-encapsulated T7-PEG- } \\
\text { DSPE-coated cationic liposomes (T7- } \\
\text { LCP/siEGFR nanoparticle) }\end{array}$ & {$[43]$} \\
\hline & Anti-miR-21, anti-miR-10b & $\begin{array}{l}\text { miR-21 and miR-10b } \\
\text { targets }\end{array}$ & $\begin{array}{l}\text { Reduction of triple negative breast cancer cells migration } \\
\text { in vitro; reduction of tumor growth in vivo }\end{array}$ & $\begin{array}{l}\text { uPA receptor-targeted antisense-loaded PLGA- } \\
\text { b-PEG polymer nanoparticles }\end{array}$ & [44] \\
\hline & $\begin{array}{l}\text { Antisense (AS) against OPN } \\
\text { and BSP }\end{array}$ & OPN and BSP & $\begin{array}{l}\text { Decrease in tumor bone metastasis incidence and size in } \\
\text { breast cancer rat model }\end{array}$ & AS-PLGA nanoparticles & [45] \\
\hline & $\begin{array}{l}\text { Anti-miR-21, 4- } \\
\text { hydroxytamoxifen }\end{array}$ & MiR-21 targets & $\begin{array}{l}\text { Antiproliferative and apoptotic effects in ER+ breast } \\
\text { cancer in vitro }\end{array}$ & $\begin{array}{l}\text { 4-OHT and anti-miR-21 co-loaded PLGA-b- } \\
\text { PEG nanoparticles }\end{array}$ & [46] \\
\hline & siRNA & BCL-XL and STAT3 & Suppression of oncogenes in HER2+ cancer cells & $\begin{array}{l}\text { pH-sensitive siRNA-loaded endosome- } \\
\text { disruptive biotinylated co-polymeric nanocarrier }\end{array}$ & [47] \\
\hline \multirow{6}{*}{$\begin{array}{c}\text { Polymeric } \\
\text { nanoparticles }\end{array}$} & MDR1-siRNA, doxorubicin & MDR1 & $\begin{array}{l}\text { Reversion of multi-drug resistance and inhibition of } \\
\text { tumor growth in vivo; reduction of doxorubicin toxicity } \\
\text { in non-target tissues }\end{array}$ & $\begin{array}{l}\text { FA-decorated PEG-b-(PCL-g-PEI)-b-PCL } \\
\text { triblock co-polymeric nanomicelles }\end{array}$ & {$[48]$} \\
\hline & BMI-1-siRNA & BMI-1 & $\begin{array}{l}\text { Reduction of BMI-1 expression in MCF- } 7 \text { breast cancer } \\
\text { cells in vitro; tumor suppression in vivo }\end{array}$ & PEI-laminarin conjugate $\mathrm{nLP} / \mathrm{siBMI}-2$ & [49] \\
\hline & ABCG2-siRNA & ABCG2 & $\begin{array}{l}\text { Gene silencing efficiency and enhancement of } \\
\text { adriamycin susceptibility in MCF-7/ADR-resistant } \\
\text { xenograft tumors in vivo }\end{array}$ & $\begin{array}{l}\text { mPEG-PLGA-PLL (PEAL) nanoparticles with } \\
\text { ultrasound-targeted microbubble destruction }\end{array}$ & {$[50]$} \\
\hline & TWIST1-siRNA & $\begin{array}{l}\text { TWIST1 and EMT- } \\
\text { related target genes }\end{array}$ & $\begin{array}{l}\text { Reduction in migration and invasion in vitro; long- } \\
\text { lasting siRNA delivery in xenograft orthotopic tumors }\end{array}$ & PAMAM dendrimer complexes & {$[51]$} \\
\hline & MDR-shRNA, doxorubicin & MDR1 & $\begin{array}{l}\text { Increase of the tumor suppression effect of doxorubicin } \\
\text { in MCF-7/adriamycin cells; reduction of doxorubicin } \\
\text { efflux }\end{array}$ & $\begin{array}{l}\text { Doxorubicin-PLGA/PEI/ MDR1-shRNA } \\
\text { nanobubbles }\end{array}$ & {$[52]$} \\
\hline & MiR-145 plasmid & MiR-145 and its targets & Cytotoxicity effect in MCF-7 cells in vitro & MiR-145 chitosan polyplex nanoparticles & {$[53]$} \\
\hline
\end{tabular}


AURKA-siRNA, paclitaxel

\section{RHOC-siRNA}

SNAIL and TWIST-siRNA, paclitaxel

VEGF-siRNA,

doxorubicin

MDR1-siRNA

\section{MYC shRNA}

IL17RB-siRNA, doxorubicin

CD73-siRNA

Anti-miR-10b LNA

\begin{abstract}
Inorganic
AKT1-siRNA
\end{abstract}

nanoparticles

Survivin-siRNA, tamoxifen

AKT-siRNA, thymoquinone

MiR-221/222 inhibitors

(miRi), paclitaxel

Hybrid nanoparticles
BCL2-siRNA, doxorubicin
AKT

AURKA

\section{RHOC GTPase}

SNAIL, TWIST

VEGF

MDR1

BCL2

MYC

IL17RB molecule CD73

BMI and other miR10b targets

AKT1

Survivin

MiR-221/222 targets

CXCR4

BMI1 and hTERT
Ectonucleotidase
Synergistic antitumor efficacy of the two drugs in MDAMB-231 breast cancer in vitro and in vivo

Inhibition of invasion, motility and migration of MDA-

MB-231 cells in vitro

Inhibition of tumor growth and metastasis in metastatic breast cancer in vivo

Antiproliferation and inhibition of tumor spheroids in

vitro; decrease in tumor microvessel density and

doxorubicin toxicity in vivo; increase in life span in mice

Overcoming multidrug resistance in adriamycin-resistant breast cancer cells in vitro and in vivo

Efficient delivery and cytotoxicity effect in MCF-7 cells in vitro

Tumor growth arrest and increased survival in mice bearing BRCA2/TP53-mut mammary tumors

Significant effect on doxorubicin -induced cytotoxicity, apoptosis induction and migration inhibition in MDAMB-361 cells in vitro

Suppression of CD73 in 4T1 breast cancer cells in vitro for immunotherapy

Induction of apoptosis, inhibition of proliferation and reduction in invasion and migration in vitro; regression of lymph node metastasis in vivo in combination with low-dose doxorubicin

Inhibition of cell cycle progression and induction of apoptosis in MCF-7 cells in vitro and in vivo

Enhancement of breast cells death in vitro

Apoptosis induction in MCF-7 cells by induction of $\mathrm{p} 53$ in vitro and in vivo; overcoming therapy resistance

Enhancement of paclitaxel efficacy on triple negative breast cancer in vitro

Reduction of CXCR4 expression in MDA-MB-231

breast cancer in vitro and in vivo

Gene silencing efficiency and tumor growth inhibition in
A-based OA-g-bPEI-conjugated redoxsensitive micellar system (HSOP) Degradable, $\mathrm{pH}$-sensitive $\beta$-cyclodextrin-based polymeric carrier (smart-anti-RHOC particles)

\section{PEI-PDHA/PEG-PDHA/PTX/siSna/siTw)}

FA-decorate $\mathrm{pH}$-responsive PHD/PPF/siVEGF nanocomplex

MDR1-targeted poly-siRNA/Thiolated glycerol chitosan nanoparticles (psi- MDR1-tGC NPs) FA-decorated hydrophilic cationic star-block terpolymer nanomicelleplexes (PGAH-bPDMAPMA)3-g-PEG

\section{PEI-grafted PGMA platform}

Carboxymethyl dextran chitosan nanoparticles (doxorubicin -siRNA-CMD-Ch NPs)

CD73-siRNA-loaded chitosan-lactate nanoparticles

Dextran coated magnetic nanoparticles (MNanti-miR-10b)

Amorphous calcium carbonate hybrid nanospheres functionalized with CaIP6 composite nanoparticles (ACC/CaIP6/siAKT1) Arginine-functionalized gold nanoparticlesstabilized capsules (NPSC/siSurv/drug) Multilamellar gold niosome containing thymoquinone and AKT-siRNA (si-RNA-NioAu- thymoquinone)

Lipid-coated calcium phosphate-polymer hybrid nanoparticles/miRi/pac

siRNA-loaded pH-responsive hybrid nanogel particles

Lipo/PEI/siRNA nano-condensate 


\section{vitro}

\section{HER2-siRNA}

VEGF-ShRNA

VEGF-siRNA, captopril

Survivin-siRNA

Anti-miR-21

RNA

nanostructures

antagomiR
HER2, delta16 HER2

VEGF

VEGF

Survivin

MiR-21 targets

MiR-205 and miR-221 targets
Reduction of tumor proliferation in trastuzumab-resistant

resonance imaging

Antiangiogenic and antitumor activity in MDA-MB-435

tumor xenografts

Survivin and stathmin

\section{doses} progression in vivo and survival advantage breast cancer in vitro and in vivo; non-toxic effect in non-tumorigenic cell lines in vitro

Inhibition of VEGF gene expression in MCF-7 cells in

vitro; potential in cancer detection by magnetic

Apoptosis induction in MCF-7 breast cancer cells in vitro

Repression of triple negative breast cancer growth at low
PEI-PEG-modified trastuzumab -conjugated mesoporous silica nanoconstructs (T-siHER2NP)

Multifunctional PEI-grafted Fe3O4@SiO2/BEGF shRNA nanocomposites

Captopril-PEI-conjugated Gold nanoparticles (siRNA/CP/GNP)

Polyacrylate-PEI-covered $\mathrm{Fe} 3 \mathrm{O} 4$ nano-sized particles (Fe3O4-PA-PEI NP)

Triple functional phi29 pRNA-3WJ

nanoparticles

Dendrimers-conjugated self-assembled RNAtriple helix hydrogel scaffolds
Inhibition of proliferation in MDA-MB-453 cells in vitro

LNA-modified chimeric aptamers

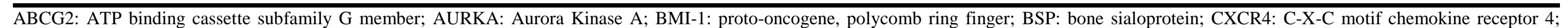

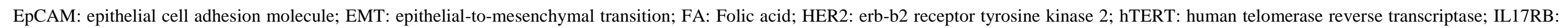

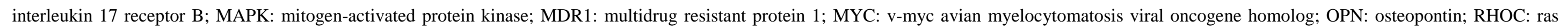

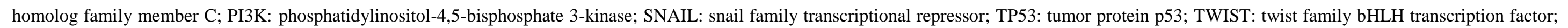
VEGF: Vascular endothelial growth factor; 
Table 2. Gene therapies in clinical trials for the treatment of breast cancer

\begin{tabular}{|c|c|c|c|c|c|c|}
\hline Vehicle and strategy & Study design & Breast cancer population & Other drugs & $\begin{array}{l}\text { Estimated } \\
\text { enrollment }\end{array}$ & Status & Study ID $^{\mathbf{a}}$ \\
\hline \multicolumn{7}{|c|}{ Viral-based strategies } \\
\hline $\begin{array}{l}\text { Oncolytic measles virus encoding thyroidal sodium } \\
\text { iodide symporter }\end{array}$ & $\begin{array}{l}\text { Open label, phase } 1 \text { safety } \\
\text { study }\end{array}$ & Metastatic breast cancer & - & 30 & Recruiting & NCT01846091 \\
\hline $\begin{array}{l}\text { Oncolytic therapy with a modified vaccinia poxvirus } \\
\text { engineered by addition of the GM-CSF gene and } \\
\text { deletion of the TK gene (JX-594) }\end{array}$ & $\begin{array}{c}\text { Randomized, open label, } \\
\text { phase } 1 / 2 \\
\text { pharmacokinetics/dynamics } \\
\text { study }\end{array}$ & Advanced breast cancer & Cyclophosphamide & 118 & Recruiting & NCT02630368 \\
\hline $\begin{array}{c}\text { Talimogene laherparepvec (HSV-1) for oncolytic } \\
\text { therapy }\end{array}$ & $\begin{array}{l}\text { Open label, phase } 1 / 2 \\
\text { efficacy study }\end{array}$ & $\begin{array}{l}\text { Triple negative breast cancer, } \\
\text { inflammatory breast cancer or } \\
\text { breast cancer with inoperable } \\
\text { recurrence }\end{array}$ & Neoadjuvant paclitaxel & 46,35 & $\begin{array}{l}\text { Not yet } \\
\text { recruiting, } \\
\text { Recruiting }\end{array}$ & $\begin{array}{l}\text { NCT02779855, } \\
\text { NCT02658812 }\end{array}$ \\
\hline $\begin{array}{l}\text { Virus-based introduction of } M D R 1 \text { into autologous } \\
\text { peripheral blood progenitor cells }\end{array}$ & Phase 2 safety/efficacy study & Metastatic breast cancer & $\begin{array}{l}\text { Methotrexate, leucovorin, 5-FU, } \\
\text { cyclophosphamide, thiotepa, } \\
\text { paclitaxel, doxorubicin }\end{array}$ & 42 & Completed & NCT00001493 \\
\hline $\begin{array}{l}\text { Suicide gene therapy with the retroviral vector Toca } \\
511 \text { encoding CD and the Toca FC prodrug 5-FC }\end{array}$ & $\begin{array}{l}\text { Open label, phase } 1 \\
\text { safety/efficacy study }\end{array}$ & $\begin{array}{l}\text { Locally advanced or metastatic } \\
\text { breast cancer }\end{array}$ & - & 26 & Recruiting & NCT02576665 \\
\hline Adenovirus-mediated delivery of TP53 & Open label, phase $1 / 2$ study & Locally advanced breast cancer & Doxorubicin, docetaxel & $20,-$ & Completed & $\begin{array}{r}\text { NCT00004038, } \\
\text { NCT00044993 }\end{array}$ \\
\hline $\begin{array}{l}\text { Retrovector bearing a dominant negative cyclin G1 } \\
\text { construct (Rexin-G) }\end{array}$ & $\begin{array}{l}\text { Open label, phase } 1 / 2 \\
\text { safety/efficacy study }\end{array}$ & $\begin{array}{c}\text { Recurrent and metastatic breast } \\
\text { cancer }\end{array}$ & - & 20 & Completed & NCT00505271 \\
\hline \multicolumn{7}{|c|}{ Non-viral based strategies } \\
\hline $\begin{array}{c}\text { Transferrin targeted cyclodextrin-containing } \\
\text { polymer carrying siRNA against RRM2 }\end{array}$ & $\begin{array}{l}\text { Open label, phase } 1 \text { safety } \\
\text { study }\end{array}$ & Refractory breast cancer & - & 24 & Completed & NCT00689065 \\
\hline \multicolumn{7}{|c|}{ Immunomodulatory strategies } \\
\hline 5T4-modified vaccinia ankara vaccine & Open label, phase 2 study & Advanced breast cancer & & - & $\begin{array}{l}\text { Not yet } \\
\text { recruiting }\end{array}$ & NCT00227474 \\
\hline
\end{tabular}


TP53-modified vaccinia ankara vaccine

Recombinant vaccinia-CEA-MUC-1-TRICOM

\section{vaccine}

Vaccination with the Venezuelan equine encephalitis

$$
\text { virus expressing HER2 }
$$

Brachyury-modified vaccinia ankara and TRICOM

$$
\text { vaccine }
$$

Vaccination with an adenovirus-encoding for HER2

Vaccination with an adenoviral vector encoding a fusion protein of human MUC-1 and CD40 ligand

Immunotherapy with an adenoviral vector engineered for the expression of human recombinant

IL-12 under the control of the RheoSwitch

Therapeutic System (RTS)

Vaccination with a plasmid DNA encoding mammaglobin-A

Vaccination with a plasmid DNA encoding CYP1B1 encapsulated in biodegradable microparticles Plasmid-based vaccination strategy targeting multiple antigens of cancer stem cells

Local injection of a DNA plasmid encoding IL-12

Vaccination with a DNA plasmid encoding $I G F B P 2$,

$$
H E R 2 \text {, and IGFIR }
$$

Vaccination with a plasmid DNA encoding HER2

Personalized polyepitope DNA vaccine
Open label, phase 1 safety

$$
\text { study }
$$

Open label, phase 1 safety

$$
\text { study }
$$

Open label, phase 1 safety

$$
\text { study }
$$

Open label, phase 1 safety

$$
\text { study }
$$

Open label, phase 1

safety/efficacy study

Open label, phase 1 safety

$$
\text { study }
$$

Open label, phase $1 / 2$ safety/efficacy study

Open label, phase 1 safety

$$
\text { study }
$$

Open label, phase 1

\begin{abstract}
safety/efficacy study
\end{abstract}
Open label, phase 1 safety

$$
\text { study }
$$

Open label, phase 1

pharmacodynamics study

Open label, phase 1 safety

$$
\text { study }
$$

Open label, phase 1

\begin{abstract}
safety/efficacy study
\end{abstract}
Open label, phase 1 safety
Triple negative breast cancer

\section{Pembrolizumab}

\section{Advanced breast cancer}

Sargramostim (GM-CSF)

51

Ongoing

HER2-positive breast cancer

Metastatic breast cancer

Locally advanced and metastatic HER2-low-

expressing breast cancer

\section{Breast cancer}

Locally advanced or metastatic breast cancer

Metastatic breast cancer

Advanced breast cancer

Advanced and recurrent HER2negative breast cancer

Triple negative breast cancer

Non-metastatic, node positive,

HER2-negative breast cancer Advanced and metastatic

HER2-positive breast cancer

Triple negative breast cancer
Veledimex

Cyclophosphamide

Sargramostim (GM-CSF)

Sargramostim (GM-CSF)

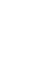

Ongoing

Ongoing

Not yet

recruiting

Recruiting

Recruiting

Completed

Completed

Recruiting

Recruiting

Recruiting

Ongoing

Recruiting
NCT02432963

NCT00088413

NCT01526473

NCT02179515

NCT02751528

NCT02140996

NCT02423902

NCT00807781

NCT00381173

NCT02157051

NCT02531425

NCT02780401

NCT00436254

NCT02348320 
Vaccination with a DNA plasmid encoding PRAME and PSMA

Immunogenic RNA vaccines

Vaccination with a HER2-targeted peptide

$$
\text { (NeuVax) }
$$

Vaccination with a HER2-targeted peptide

$$
\text { (NeuVax) }
$$

Vaccination with a HER2-targeted peptide (AE37)

Vaccination with four tumor-associated peptides

Vaccination with four tumor-associated peptides

$$
\text { (PVX-410) }
$$

Combination of two chimeric (trastuzumab-like and pertuzumab-like) HER-2 B cell peptide vaccine linked to a promiscuous $\mathrm{T}$ cell epitope from the measles virus fusion protein

Vaccination with autologous breast cancer cells

$$
\text { engineered to secrete GM-CSF }
$$

Vaccination with a CD80-modified, devitalized breast cancer cell line (KS24.22)

Vaccination with four tumor-associated peptides Anti-TP53 TCR-gene engineered lymphocytes and autologous dendritic cell-adenovirus TP53 vaccine
Randomized, open label,

phase 1 safety/efficacy study

Open label, phase 1 safety/efficacy study

Triple negative breast cancer

Low and intermediate HER2-

Randomized, single blind, phase 2 efficacy study

expressing breast cancer, highrisk HER2-positive breast

\section{cancer}

Randomized, double blind, phase 3 safety/efficacy study

Randomized, single blind, phase 2 efficacy study

Open label, phase 1 safety/efficacy study Open label, phase 1 safety/efficacy study

\section{Open label, phase 1} safety/efficacy study

Open label, phase $1 / 2$ safety/efficacy study Open label, phase 1 safety/efficacy study Open label, phase 1 safety/efficacy study

Open label, phase 2 study
Low and intermediate HER2expressing breast cancer

Node-positive and high-risk node-negative HER2-positive breast cancer

Triple negative breast cancer

Triple negative breast cancer

Advanced HER2-positive breast cancer

Metastatic breast cancer, Operable Breast Cancer

Metastatic breast cancer

Triple negative breast cancer

Progressive or recurrent metastatic breast cancer
Sargramostim (GM-CSF),

$$
\text { trastuzumab }
$$

Sargramostim (GM-CSF)

Sargramostim (GM-CSF)

Durvalumab

Durvalumab$$
-
$$$$
-
$$

Durvalumab

Cyclophosphamide, fludarabine, aldesleukin (IL-2), filgrastim (G-
20,20

20
Recruiting

Recruiting

\section{NCT02316457}

NCT01570036, NCT02297698

Ongoing NCT01479244

Ongoing NCT00524277

Recruiting NCT02826434

Recruiting NCT02826434

Recruiting NCT01376505

NCT00317603, NCT00880464

Completed NCT01127074

Recruiting NCT02826434

Completed NCT00704938 
Treatment with a recombinant fusion protein of IL-2 linked to a single-chain TCR domain targeting TP53 Immunotherapy with modified TCR targeting NY-

\section{ESO-1 tumor antigen}

Immunotherapy with modified TCR targeting CEA

$$
\text { tumor antigen }
$$

Engineered CAR T cells targeting mesothelin

\section{Engineered CAR T cells targeting CD133}

Engineered CAR T cells targeting EpCAM

Engineered CAR T cells targeting ROR1

\section{Engineered CAR T cells targeting HER2}

Engineered CAR T cells targeting MET proto-

$$
\text { oncogene }
$$

Vaccination with autologous DCs infected with an adenovirus expressing $H E R-2$

Vaccination with adenovirus-TP53 transduced DCs

Vaccination with adenovirus-HER2 transduced DCs
Open label, phase 1 safety/efficacy study Open label, phase $1 / 2$ safety/efficacy study

Open label, phase 1 safety/efficacy study

Open label, phase 1 safety/efficacy study

Open label, phase 1 safety/efficacy study

Open label, phase 1 study

Open label, phase 1 safety study

Open label, phase $1 / 2$ safety/efficacy study

Phase 1/2 safety/efficacy study

Open label, phase 1 safety study

Open label, phase $1 / 2$ safety/efficacy study

Open label, phase 1 safety study
Metastatic breast cancer

NY-ESO-1 expressing breast cancer

Metastatic breast cancer,

relapse or refractory breast

$$
\text { cancer }
$$

Relapsed and refractory

advanced triple negative breast cancer, metastatic HER2-

negative breast cancer

Relapsed and refractory

advanced triple negative breast

$$
\text { cancer }
$$

Recurrent breast cancer

Recurrent and metastatic breast cancer, triple negative breast

$$
\text { cancer }
$$

Recurrent, metastatic or refractory HER2-positive

$$
\text { breast cancer }
$$

Operable triple negative breast

$$
\text { cancer }
$$

HER2-positive breast cancer

Recurrent and advanced breast cancer

HER2-positive breast cancer

$$
\text { aldesleukin (IL-2) }
$$

Cyclophosphamide
Cyclophosphamide, fludarabine,

43,36

Recruiting

60,75

Ongoing/Recr

uiting

20,24

Recruiting

NCT02580747, NCT02792114

Recruiting

Recruiting

Recruiting

60,60

Recruiting

NCT02547961, NCT02713984

Ongoing

NCT01837602

Completed

NCT00197522

$44 \quad$ Ongoing NCT01042535

Recruiting

NCT01730118 
Locally advanced, triple-
Vaccination with cyclin B1/WT-1/CEF pool-loaded

\section{DCs}

Vaccination with autologous DCs pulsed with onco-

$$
\text { peptides }
$$

Vaccination with DCs incorporating tumor blood

$$
\text { vessel antigen-derived peptides }
$$

Vaccination with DCs transfected with survivin,
Open label, phase $1 / 2$

safety/efficacy study

Open label, phase $1 / 2$

safety/efficacy study

Open label, phase 1 safety

study

Open label, phase 1 safety negative breast cancer or ER-

positive, HER2-negative breast

cancer

Metastatic breast cancer

Metastatic breast cancer

Metastatic breast cancer
NCT02018458

\section{hTERT and TP53}

study

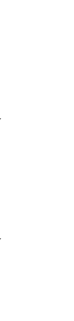

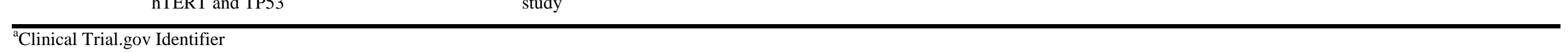

${ }^{\mathrm{a} C l i n i c a l}$ Trial.gov Identifier

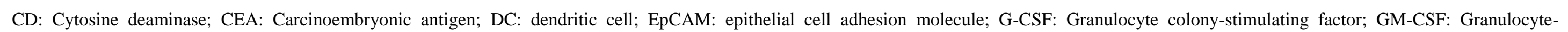

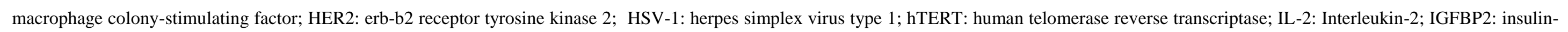




thymidine kinase; WT1: Wilms tumor protein 1; 5-FC: 5-fluorocytosine; 5-FU: 5-fluorouracil; 5T4: Tumor antigen 5T4. 


\section{Figure legends}

Figure 1. Schematic representation of multifunctional nanovectors for gene delivery. Multiple non-viral delivery systems have been developed for gene therapy. Different lipid formulations and functionalization strategies have been employed to improve the safety, stability and efficiency of nanovectors. The opportunity to load many types of cargos within the core, as well as to functionalize the outer layers, makes nanoparticles a versatile system for gene therapy.

Figure 2: Overview of the major immune-based approaches for cancer gene therapy. Autologous T cells can be genetically modified to express a novel TCR or a CAR to specifically recognize a tumor-associated antigen. Therapeutic cancer vaccines can derive from whole tumor cells, protein antigens, peptides, DNA, or dendritic cells, and are designed to potentiate anti-cancer immune response. These strategies can be administer in combination with immunostimulatory cytokines to boost anti-tumor activity.

CAR: Chimeric antigen receptor; HLA: human leucocyte antigen; IL-2: Interleukin-2; G-CSF: Granulocyte colony-stimulating factor; GM-CSF: Granulocyte-macrophage colony-stimulating factor; TCR: T cell receptor. 









